\title{
Olanzapine Reduces Craving for Alcohol: A DRD4 VNTR Polymorphism by Pharmacotherapy Interaction
}

\author{
Kent E Hutchison*,', Angela Wooden', Robert M Swift ${ }^{2}$, Andrew Smolen', John McGeary', Lawrence Adler ${ }^{3}$ \\ and Lyndee Paris' \\ 'Department of Psychology, University of Colorado at Boulder, Boulder, CO, USA; ${ }^{2}$ Providence Veteran Affairs Medical Center and Center for \\ Alcohol and Addiction Studies at Brown University, USA; ${ }^{3}$ University of Colorado Health Sciences Center, USA
}

\begin{abstract}
Separate investigations have suggested that olanzapine, a D4 antagonist, decreases craving after a priming dose of alcohol and that the DRD4 variable number of tandem repeats (VNTR) polymorphism influences the expression of craving after a priming dose of alcohol. The present study tested the hypothesis that olanzapine may be differentially effective at reducing cue-elicited craving based on individual differences in DRD4 VNTR in a sample of heavy social drinkers. Participants were randomly assigned to receive olanzapine (5 mg) or a control medication (cyproheptadine, $4 \mathrm{mg}$ ) prior to consuming three alcoholic drinks. Participants completed subjective measures of craving and euphoria after each drink. Participants who were homozygous or heterozygous for the 7 (or longer) repeat allele of the DRD4 VNTR were classified as DRD4 L, while the other participants were classified as DRD4 S. The findings indicated that olanzapine reduces craving for alcohol at baseline for both DRD4 S and DRD4 L individuals, but only reduces craving after exposure to alcohol cues and after a priming dose of alcohol for DRD4 $\mathrm{L}$ individuals.

Neuropsychopharmacology (2003) 28, I 882- I 888, advance online publication, 23 July 2003; doi: I 0. I 038/sj.npp. I 300264
\end{abstract}

Keywords: alcohol; craving; olanzapine; gene; DRD4

\section{INTRODUCTION}

To date, only two pharmacotherapies, disulfram and naltrexone, have been approved for the treatment of alcohol dependence, and both these medications are only modestly effective (Swift, 1999). The need for new, more effective, pharmacological interventions has prompted the National Institute on Alcoholism and Alcohol Abuse (NIAAA) and others to initiate programs to test and develop treatments that target specific mechanisms that play an important role in the etiology of alcohol dependence and relapse, such as craving and protracted withdrawal. The action of alcohol and other drugs on the mesolimbic dopamine pathways is thought to be an important mechanism in the etiology of alcohol and drug dependence and more specifically, the development of intense craving and loss of control (Berridge and Robinson, 1998; Robinson and Berridge, 1993; Wise, 1988). Formally described as 'an incentive sensitization' model of craving, this perspective on craving proposes that mesolimbic dopamine activation influences the motivational and appetitive properties of alcohol and

\footnotetext{
*Correspondence: Dr KE Hutchison, Department of Psychology, University of Colorado, Muenzinger Psychology Building, Campus Box 345, Boulder, CO 80309-0345, USA, Tel: + I 303492 2967, Fax: + I 303492 3298, E-mail: KentH@psych.colorado.edu

Received 02 January 2003; revised 03 May 2003; accepted 08 May 2003

Online publication: 10 June 2003 at http://www.acnp.org/citations/ Npp06 I003030004/default.pdf
}

drugs by controlling the attribution of incentive salience to neural representations of alcohol and drug-related stimuli (Berridge and Robinson, 1998; Robinson and Berridge, 1993; Wise, 1988). The acquisition and sensitization of incentive salience (ie craving) for drugs is produced by repeated drug ingestion and the expression of incentive salience can be activated by the release of dopamine that is initiated in response to drug cues or priming doses of the drug itself (de Wit, 1996). Previous studies with humans have provided direct evidence that alcohol and alcohol cues increase activation in the same neural substrates that serve the process of incentive sensitization in humans (Modell and Mountz, 1995).

Recent research with humans has suggested that the sight and smell of alcohol, as well as consumption of small doses of alcohol, increases craving relative to consumption of nonalcoholic beverages and that this craving is attenuated by olanzapine, an atypical dopamine antagonist that acts on $\mathrm{D}_{4}$ and $\mathrm{D}_{2}$ receptors (Hutchison et al, 2001). The study with olanzapine is consistent with previous work indicating that consumption of alcohol increases craving and that a traditional dopamine antagonist (ie haloperidol), that acts on $\mathrm{D}_{2}$ and $\mathrm{D}_{4}$ receptors, reduces alcohol-elicited craving (Modell et al, 1993). In addition to these laboratory studies on dopamine antagonists and alcohol craving, recent clinical reports have suggested that clozapine, a $\mathrm{D}_{4}$ receptor antagonist that is similar to olanzapine, reduces substance abuse among patients with comorbid substance abuse/ dependence (Green et al, 1999) and specifically, alcohol use 
(Drake et al, 2000). Thus, research with humans suggests that selective targeting of dopamine receptor subtypes may be useful for developing pharmacotherapies that reduce craving.

Not only is it important to target specific mechanisms with new treatments, it is equally important to elucidate individual differences in these mechanisms that are likely to predict treatment outcome (Gordis, 2000). Individual differences in the development of loss of control drinking and the ability to stop drinking are likely to be related to genetic factors that influence the effects of alcohol and alcohol cues on mesolimbic dopamine activation and craving. A recent investigation was conducted to examine whether the DRD4 variable number of tandem repeats (VNTR) polymorphism moderated the effects of alcohol on craving and other variables (Hutchison et al, 2002a). Based on our previous studies and molecular work suggesting that the 7 repeat allele is distinct from the 2-6 repeat alleles (Ding et al, 2002), participants in this study were classified as DRD4 L (ie homozygous or heterozygous for an allele $\geqslant 7$ repeats; S/L or L/L) or were classified as DRD4 S (ie both alleles $<7$ repeats; S/S). It was hypothesized that if craving after the consumption of alcohol is related to the functional differences in the $\mathrm{D}_{4}$ receptor expressed by the DRD4 VNTR polymorphism, DRD4 L participants should show a differential craving response after alcohol consumption as compared to DRD4 S participants. The results suggested that DRD4 L participants demonstrated significantly higher craving after consumption of alcohol as compared to the control beverage, while DRD4 S participants did not.

To replicate and extend our previous results with olanzapine and our previous study on the DRD4 VNTR, the present study was designed to examine whether olanzapine $(5 \mathrm{mg})$ reduced craving as compared to cyproheptadine ( $4 \mathrm{mg})$, which was conceptualized as an active control medication. Olanzapine is a potent $\mathrm{D}_{4}, \mathrm{D}_{2}, 5-\mathrm{HT}_{2}$, and $\mathrm{H}_{1}$ antagonist that may cause drowsiness, whereas cyproheptadine is also a powerful $5-\mathrm{HT}_{2}$ and $\mathrm{H}_{1}$ antagonist that may cause drowsiness. Thus, cyproheptadine represents an active control that can be used to isolate the effect of olanzapine better and exclude alternative explanations (eg drowsiness as a causal agent). It is important to note that there are very few published studies (to our knowledge) that have used such a stringent experimental control in a test of a pharmacological agent that targets alcohol craving. If dopamine receptors mediate the effect of alcohol cues and priming doses of alcohol on craving, we would expect olanzapine to attenuate craving relative to the active control medication. Likewise, if the 7 repeat allele of the DRD4 VNTR is a vulnerability marker in terms of the effect of alcohol on dopamine activity at the $\mathrm{D}_{4}$ receptor and craving after alcohol consumption, we would expect olanzapine to be most effective among individuals with the 7 repeat allele (ie the DRD4 L individuals).

\section{METHODS}

\section{Participants}

The study was approved by the University of Colorado Human Research Committee, and all subjects provided written informed consent after receiving a full explanation of the study. All female subjects tested negative for pregnancy prior to participation, all subjects were required to have a blood alcohol concentration of zero before each session, and all subjects were required to be in excellent health, as indicated by a thorough medical screening (eg medical exam, CBC, EKG, LFTs), to ensure that there were no contraindications for the use of the study medications or alcohol. Subjects were also excluded if they reported a psychiatric diagnosis or psychiatric treatment, reported the use of illicit drugs other than marijuana, or tested positive for the use of illicit drugs other than marijuana. Additional inclusionary criteria included scoring 8 or higher on the Alcohol Use Disorders Identification Test (AUDIT), having a preference for beer, consuming alcohol at least twice per week, and consuming an average of three drinks per occasion (two for women). Despite the potential for limited generalizability, these inclusionary criteria were designed specifically to generate a younger, healthier sample because of concerns about possible side effects with olanzapine.

The research participants were screened medically at the University of Colorado at Boulder General Clinical Research Center. Of the 75 individuals who were approved for the study, six did not complete the study due to drowsiness or nonspecific reasons (five in the olanzapine condition and one in the cyproheptadine condition). In addition, one participant did not complete the craving assessments and one participant was not compliant with the medication, leaving 67 individuals (24 women, 64 Caucasian, one African-American, two Asians, two Hispanics, and one of other ethnicity) who completed the study.

\section{Medication Administration Procedures}

Volunteers were randomly assigned to receive either olanzapine $(5 \mathrm{mg})$ or cyproheptadine $(4 \mathrm{mg}) .{ }^{1}$ All subjects were specifically instructed not to drink once they started the study. A total of 37 participants received olanzapine and 30 received cyproheptadine. A previous study suggested that olanzapine attenuated alcohol-elicited craving as compared to a placebo control (Hutchison et al, 2001). As olanzapine has moderate affinity for receptors other than $\mathrm{D}_{2}$ and $\mathrm{D}_{4}$ receptors (eg mainly $5-\mathrm{HT}_{2}, \mathrm{H}_{1-2}, \mathrm{M}_{1-4}$, less so for $5-\mathrm{HT}_{3}$ and $\mathrm{GABA}$ ), it was not clear in that study whether the effects of olanzapine were due to dopamine antagonism, 5$\mathrm{HT}_{2}$ antagonism, or sedation via histamine and muscarinic antagonism. Cyproheptadine was used as a control medication in the present experiment because it is also effective in antagonizing $5-\mathrm{HT}_{2}$ receptors in humans but does not block dopamine receptors (Kapur et al, 1997). Thus, the experimental design allowed us to exclude the action of olanzapine at $5-\mathrm{HT}_{2}$ receptors, and more generally, the sedative effects of olanzapine as explanations for the reduction in alcohol-elicited craving in our previously published study.

The participants and the experimenter were blind to the medication condition. Participants were instructed to take

\footnotetext{
${ }^{1}$ This study was originally designed to include a third control medication (diphenhydramine, $25 \mathrm{mg}$ ). However, the diphenhydramine caused significantly greater sedation as compared to the olanzapine and cyproheptadine and was dropped from the study design. There were not enough DRD4 $\mathrm{L}$ individuals to include the diphenhydramine subjects in the analyses.
} 
five consecutive doses of the study medication at intervals roughly equal to the half-life for the medication. Participants who were assigned to the olanzapine group took the medication at bedtime every day for 4 days and took the last dose $6 \mathrm{~h}$ prior to the experimental session. Participants were instructed to take the medication prior to going to bed in order to diminish the impact of any drowsiness experienced as a result of the olanzapine. Participants in the cyproheptadine group took their medication every $8 \mathrm{~h}$ and took the last dose $2 \mathrm{~h}$ prior to the experimental session. Participants called the experimental office after taking each dose to confirm when it was taken and to report whether they had experienced any adverse effects from the previous dose. To ensure that the primary experimenter was blind to the medication condition, a second experimenter was responsible for the random assignment. The second experimenter also provided the medication instructions to the subject in a sealed envelope and recorded the voice mails from subjects confirming that they took the medication.

In order to confirm that participants took the medication, the medications were packed into an opaque capsule with $50 \mathrm{mg}$ of riboflavin. A urine sample was collected on the morning of the experimental session. The urine sample was tested for riboflavin content by examining it under an ultraviolet light; a procedure that makes the riboflavin detectable (Del Boca et al, 1996). As noted above, only one participant was not compliant and these data were not included in the analyses. At the end of each experimental session, participants were asked which medication they believed that they had received.

\section{Experimental Procedures}

At the beginning of the session, participants were seated at a desk and instructed to relax in order to allow them to habituate to the laboratory before completing baseline measures of craving, stimulation, and sedation (see description of measures below). Participants were then exposed to alcohol cues. The alcohol cues consisted of each individual's favorite beer in its commercially labeled container, which was placed in front of them with a glass. The participants were then instructed to pour the beverage into the glass, lift the glass to their face, smell the beverage, and let it touch their lips. After the exposure period, the cue was removed and the participants were instructed to complete another battery of assessments.

Participants consumed alcohol after the cue exposure period. To maintain consistency in the amount of alcohol consumed, each subject consumed the same alcoholic beverage (a high alcohol content beer) consistent with our previous studies (Hutchison et al, 2001, 2002a,b). The amount of alcohol is determined by a nomogram that factors in the influence of height, weight, and gender to achieve a desired peak blood alcohol level (BAL) of 0.06 (Watson, 1989). Each dose contained $0.15 \mathrm{~g} / \mathrm{kg}$ doses of ethyl alcohol $(0.11 \mathrm{~g} / \mathrm{kg}$ for females). Participants consumed each dose at an even pace over $2 \mathrm{~min}$ and then relaxed for 3 min before beginning a battery of measures (described below). At $20 \mathrm{~min}$ after beginning the drink, participants were instructed to consume the second drink following the same procedures. The third and final drink was consumed at $20 \mathrm{~min}$ after the second drink. A final self-report assessment was conducted 20 min after the third drink.

\section{Individual Difference and Experimental Measures}

Prior to the experimental session, participants completed a battery of individual difference measures that included demographic questions as well as measures of drinking behavior and personality.

The Alcohol Dependence Scale (ADS). This measure is used to assess the severity of alcohol-related problems (Skinner and Allen, 1982).

A 30-day Time Line Follow Back procedure (TLFB; Sobell and Sobell, 1980). This measure was used to measure the quantity and frequency of drinking in the 30 days prior to the experiment.

Years of sustained drinking. This variable was assessed by the item, 'How many years have you been drinking the quantity of alcohol per week that you are drinking now?'

Alcohol Urge Questionnaire (AUQ). The AUQ was used to assess urge to drink. The AUQ consists of eight items related to urge to drink that are rated on a 7-point Likert scale with the extremes anchored by 'Strongly Disagree' and 'Strong Agree.' The AUQ has demonstrated internal consistency and reliability (Bohn et al, 1995).

Biphasic Alcohol Effects Scale (BAES). The BAES was used to collect information on changes in self-reported stimulation after alcohol administration. The BAES has previously demonstrated reliability and validity in investigations of the stimulatory and sedative effects of alcohol (Martin et al, 1993) and for assessing medication effects (Swift et al, 1994). The stimulation subscale consists of seven adjectives associated with stimulation rated from 0 (least) to 10 (most), while the sedation subscale consists of seven items associated with sedation rated from 0 (least) to 10 (most).

Drink ratings. Participants were asked to make ratings after each drink regarding how high they were feeling ('How high (as in drug high) do you feel?' anchored by 'Do not feel high at all' and 'feel extremely high') on a 100-point scale.

Side effect checklist. This measure was used to assess the existence of 24 side effects (scored as present or absent) at the beginning and end of the experimental session.

Check on blind. Participants indicated which of the medications that they believed they received.

\section{DNA Extraction and Genetic Analysis}

Genomic DNA was collected at baseline and isolated from buccal cells using published procedures (Lench et al, 1988). The $48 \mathrm{bp}$ VNTR in the third exon of the DRD4 was assayed using previously reported methods (Sander et al, 1997). The primer sequences were forward, 5'-AGGACCCTCATGGCCTTG-3' (fluorescently labeled), and reverse, $5^{\prime}$ GCGACTACGTGGTCTACTCG-3' (Lichter et al, 1993). Participants were classified as DRD4 L (ie homozygous or heterozygous for an allele $\geqslant 7$ repeats; $S / L$ or $L / L$ ) or were classified as DRD4 S (ie both alleles $<7$ repeats; S/S). Of the 67 participants, two were homozygous for the 7 repeat allele. There were not enough participants to conduct a meaningful statistical analysis of the homozygous individuals independent of those who were heterozygous for the 7 repeat allele. 
Table I Pretest Differences Between the Medication Groups

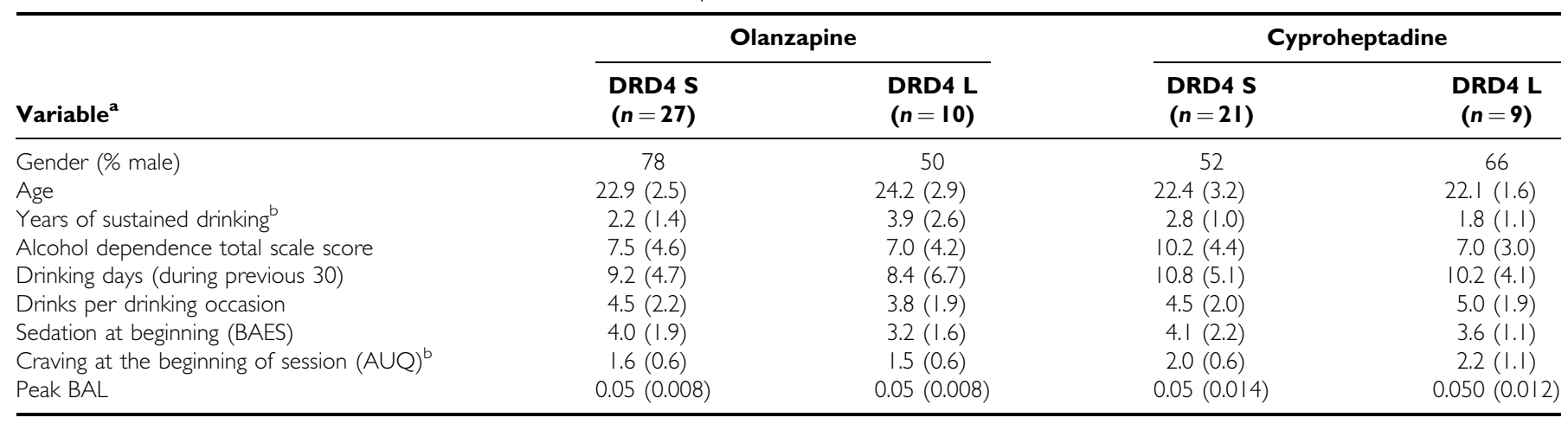

astandard deviations appear in parentheses below the means of continuous variables.

bignificant Medication by DRD4 interaction $(p<0.05)$.

\section{RESULTS}

\section{Overview}

To assess the integrity of the blind, a $\chi^{2}$ test was performed on the item asking participants to guess which medication they received. The test was nonsignificant $(p>0.10)$. Only 12 of the 37 participants who received olanzapine guessed correctly, suggesting that the blind worked quite well.

To determine whether the medication groups differed on baseline variables, a series of analyses of variance (ANOVA) tests were conducted to determine whether the Medication by DRD4 groups differed at baseline (see Table 1 for the means and standard deviations for the Medication by DRD4 groups). Analyses of the baseline data suggested that the cyproheptadine-treated group with the 7 repeat allele had not been drinking as long as the other groups. ${ }^{2}$ It should also be noted that sedation was equivalent across groups, while baseline craving was significantly lower for the olanzapine-treated groups. Finally, a repeated measures ANOVA was conducted to determine whether the groups differed in terms of BALs after each drink. There were no differences in BAL (see Table 1 for peak BAL).

To assess whether side effects differed across medication groups, $\chi^{2}$ tests were conducted on each of 24 items describing typical side effects. There was a trend for greater appetite $(p=0.055)$ and greater confusion $(p=0.059)$ among participants receiving olanzapine, but no other significant differences $(p>0.10)$. Finally, smoking status did not differ between the medication groups. Three individuals who received cyproheptadine were current smokers and two individuals who received olanzapine were current smokers.

\section{Cue-Elicited Craving}

To analyze reactivity to alcohol cues, a series of $2 \times 2 \times 2$ mixed design ANOVAs were conducted where Cue (baseline $v s$ alcohol cues) was a two-level within-subject factor, Medication (olanzapine or cyproheptadine) was a two-level

\footnotetext{
${ }^{2}$ When the analyses were reconducted with the number of years of sustained drinking as a covariate, the Medication by DRD4 interaction effect sizes were all greater. We decided to present the main analyses without this covariate because it represents a more conservative perspective on the effects noted in the present study and simplifies the interpretation.
}

between-subject factor, and DRD4 (DRD4 L vs DRD4 S) was a two-level between-subject factor.

The analyses revealed a significant main effect for Medication, $\mathrm{F}(1,63)=4.88, p<0.05$, a significant Medication by DRD4 interaction, $\mathrm{F}(1,63)=4.58, p<0.05$, and a significant Medication by DRD4 by Cue interaction, $\mathrm{F}(1,63)=8.54, p<0.01$. Olanzapine reduced craving among the DRD4 L participants at baseline and after exposure to alcohol cues. Olanzapine also attenuated craving among the DRD4 S participants at baseline. There were no significant effects for the medication on craving after alcohol cue exposure among the DRD4 $\mathrm{S}$ individuals, suggesting that olanzapine was not effective at reducing cue-elicited craving among the DRD4 S individuals (see Figure 1).

\section{Effects of Alcohol}

To address reactivity to priming doses of alcohol, a series of $3 \times 2 \times 2$ mixed design ANOVAs were conducted where type of Trial (first, second, or third drink) was a three-level within-subject factor, Medication (olanzapine vs cyproheptadine) was a two-level between-subject factor, and DRD4 (DRD4 L vs DRD4 S) was a two-level between-subject factor. The dependent variables examined were the craving score on the AUQ, feeling 'high', and the stimulation and sedation scales of the BAES.

\section{Craving (AUQ)}

There was a main effect for Medication, $F(1,63)=7.32$, $p<0.01$, but no main effect for the DRD4 VNTR or Trial


Figure I Mean craving (AUQ) scores at baseline and after exposure to alcohol cues. Analyses indicated a Medication by DRD4 by Trial interaction $(p<0.05)$ such that olanzapine significantly reduced craving at baseline and after cue exposure among the DRD4 $L$ individuals and reduced craving at baseline among the DRD4 S individuals. Olanzapine did not reduce cueelicited craving among the DRD4 S individuals. 

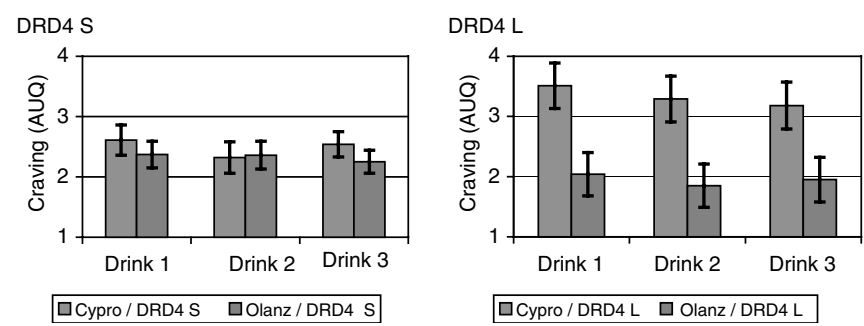

Figure 2 Mean craving (AUQ) scores and standard errors after each drink. Analyses indicated that olanzapine significantly reduced craving for alcohol after consumption $(p<0.05)$ among the DRD4 $L$ individuals, but not the DRD4 S individuals.
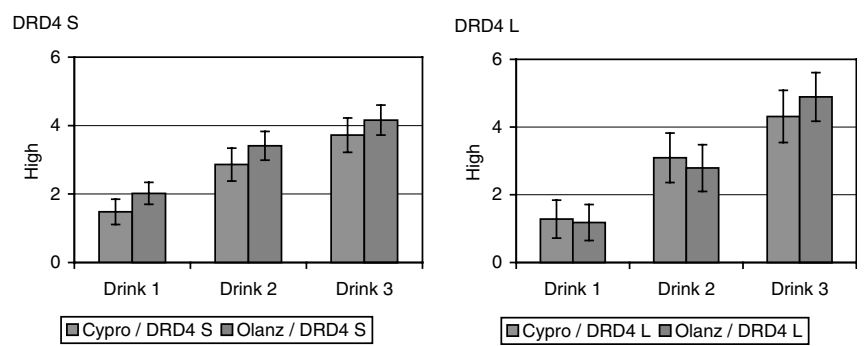

Figure 3 Mean subjective high and standard errors after each drink for the DRD4 $S$ and DRD4 L groups. Analyses indicated that alcohol significantly increased subjective high across drinks $(p<0.0 \mathrm{I})$ and indicated that this increase was greater among the DRD4 L individuals $(p<0.05)$.

$(p>0.05)$. The analyses also revealed a significant Medication by DRD4 interaction, $\mathrm{F}(1,63)=4.51, p<0.05$. As can be seen in Figure $2 a$ and $b$, olanzapine significantly reduced craving after alcohol consumption among DRD4 $\mathrm{L}$ individuals $(p<0.05)$. Olanzapine did not reduce craving after alcohol consumption among the DRD4 S individuals. To control for the effects of olanzapine on craving at baseline, the analyses were repeated with baseline craving entered as a covariate. In this respect, the analysis reflected the specific effect of the medication on craving after the consumption of alcohol, rather than the more general effect of olanzapine on baseline craving. The Medication by DRD4 interaction was still significant $(p<0.05)$, even when controlling for the effect of olanzapine on craving at baseline.

\section{Feeling High}

There was no main effect of Medication or DRD4 $(p>0.05)$. There was a main effect for Trial, $\mathrm{F}(2,126)=087.45$, $p<0.01$, and a significant DRD4 by Trial interaction, $\mathrm{F}(2,126)=4.10, p<0.05$, such that DRD4 L indicated that their 'high' increased more across trials than DRD4 S individuals (see Figure $3 a$ and $b$ ). The three-way interaction was not significant.

\section{Stimulation and Sedation (BAES)}

With respect to stimulation and sedation, there were no significant main effects or interactions $(p>0.05)$.

\section{DISCUSSION}

The findings of the present study indicate that olanzapine, relative to the control medication, reduces craving for alcohol at baseline for both DRD4 S and DRD4 L individuals, but only reduces craving after exposure to alcohol cues and after a priming dose of alcohol for DRD4 L individuals. Thus, the findings suggest a pharmacotherapy by gene interaction such that olanzapine is most effective at reducing cue-elicited craving and craving after a priming dose of alcohol among DRD4 L individuals. These findings provide a convincing replication of our previous report suggesting that olanzapine reduces craving for alcohol (Hutchison et al, 2001) and extends our previous work with the DRD4 VNTR (Hutchison et al, 2002a) by indicating that the DRD4 VNTR moderates the effectiveness of olanzapine in terms of attenuating craving. Furthermore, olanzapine appeared to have no effect on measures of reward (eg 'high' and stimulation), consistent with our previous study (Hutchison et al, 2001). Unlike our previous work with the DRD4 VNTR, the DRD4 L individuals reported greater increases in subjective high across drinks, suggesting that these individuals may experience greater euphoria and reward during the consumption of alcohol. Given the inconsistency with our previous work, this finding should be viewed with caution.

It is highly unlikely that third variables may have biased the findings of the present study. There were no significant differences in the ages, gender, ethnicity, or drinking patterns during the previous 30 days, suggesting that differences in these variables did not account for the differences in alcohol-elicited craving. The threat of population stratification is also unlikely. Population stratification is primarily a threat when the effect sizes are small and samples consist primarily of two distinct populations (Wacholder et al, 2000; Hutchison et al, in press). Given that the sample was $90 \%$ Caucasian (mixed European ancestry), population stratification is an unlikely threat to the internal validity of the study. Even if the sample consisted of two distinct Caucasian subpopulations with different allele frequencies, there would also need to be a significant difference in craving across these subpopulations in order for stratification to threaten the internal validity of these findings, and there is no evidence to suggest such differences across European subpopulations. Although population stratification is not much of a threat in the present study, further research with additional ethnic groups will be necessary to determine if these findings generalize to other populations. In addition, future work with the DRD4 VNTR will determine how consistent this effect is across studies on alcohol craving and, more generally, studies of appetitive motivation. To date, studies have suggested that the DRD4 VNTR may influence appetitive motivation for alcohol (Hutchison et al, 2002a), tobacco (Hutchison et al, 2002b), and food (Sobik et al, under review).

To preclude any concerns over the lack of a priori predictions regarding the interaction with the DRD4 VNTR, the present study was specifically designed to reflect a biological framework (ie the dopamine system and specifically the D4 receptor) that connects the behavior (ie craving in a high-risk situation) to a specific medication (ie 
olanzapine) and a moderating genetic factor (ie the DRD4 VNTR polymorphism). Given the design of the study, alternative explanations for the findings involving 5-HT2 receptors or sedation are less problematic. In this respect, the design resolves some of the interpretative issues in our previous study with olanzapine (Hutchison et al, 2001). It should be noted that we cannot conclude that other pharmacological properties of olanzapine did not influence the findings. For example, the 5-HT3 antagonist ondansetron may reduce alcohol consumption and craving among subpopulations of alcoholics (Johnson et al, 2002) and olanzapine also acts as a 5-HT3 antagonist with an affinity similar to ondansetron. However, a 5-HT3 effect cannot account for the findings in the present study given that other D4 antagonists (eg clozapine) reduce substance use and given the interaction with the DRD4 VNTR. It is more likely that the action of olanzapine at $5-\mathrm{HT} 3$ receptors may influence craving above and beyond the action of olanzapine at dopamine receptors. Thus, the 5-HT3 effect of olanzapine may provide additional benefits beyond a dopaminergic effect. If this were the case, olanzapine would be expected to show superior efficacy in a direct comparison with ondansetron.

One of the priorities of NIAAA and the field in general is advancing candidate medications that target craving, among other mechanisms, and identifying individual differences that may predict the effects of new medications on this target. The findings from the current study suggest that a specific biological mechanism (ie the effect of alcohol on mesolimbic dopamine and the $\mathrm{D}_{4}$ receptor) is critical to one of the primary behavioral components of alcohol abuse and dependence (eg craving). Not only have preliminary studies identified olanzapine as a medication that acts on this target, preliminary studies have also suggested that the DRD4 VNTR may be related to individual differences in vulnerability to craving and the effects of olanzapine on craving. Thus, the findings from the present study also suggest that DRD4 L individuals may be particularly susceptible to the effect of alcohol cues and priming doses of alcohol on these dopamine receptors and that these individuals may benefit most from biological interventions that reduce the expression of incentive salience (ie medications that target dopamine receptors). Medications that target the $\mathrm{D}_{4}$ receptor and genetic factors that influence the function of $\mathrm{D}_{4}$ receptors may prove to play an important role in the treatment of alcohol dependence.

Despite this preliminary work, it has yet to be determined whether the effect of olanzapine on craving will translate into a reduction in alcohol use behavior and alcohol dependence symptoms among alcoholics. Clearly, the sample is one of the limitations of the present study. It is unclear whether the effects of olanzapine in a sample of heavy social drinkers will generalize to a sample of treatment-seeking alcoholics. To establish whether olanzapine or a similar medication may enhance abstinence, future research should examine the relative effectiveness of olanzapine in a sample of alcoholics and examine how well the medication is tolerated in this population. Even if olanzapine is not well tolerated, future studies may examine the utility of other $\mathrm{D}_{4}$ antagonists that have been recently tested in humans.

\section{ACKNOWLEDGEMENTS}

This research was supported by grants from the National Institute on Alcoholism and Alcohol Abuse (R01AA11473) and the General Clinical Research Center Program of the National Center for Research Resources, National Institutes of Health (M01-RR00051).

\section{REFERENCES}

Berridge KC, Robinson TE (1998). What is the role of dopamine in reward: hedonic impact, reward learning, or incentive salience? Brain Res Brain Res Rev 28: 309-369.

Bohn MJ, Krahn DD, Staehler BA (1995). Development and initial validation of a measure of drinking urges in abstinent alcoholics. Alcohol Clin Exp Res 19: 600-606.

de Wit H (1996). Priming effects with drugs and other reinforcers. Exp Clin Psychopharmacol 4: 5-10.

Del Boca FK, Kranzler HR, Brown J, Korner PF (1996). Assessment of medication compliance in alcoholics through UV light detection of a riboflavin tracer. Alcohol Clin Exp Res 20: 1412-1417.

Ding Y-C, Chi H-C, Grady DL, Morishima A, Kidd JR, Kidd KK et al (2002). Evidence of positive selection acting at the human dopamine receptor D4 gene locus. Proc Natl Acad Sci 99: 309-314.

Drake RE, Xie H, McHugo GJ, Green AI (2000). The effects of clozapine on alcohol and drug use disorders among patients with schizophrenia. Schizophr Bull 26: 441-449.

Gordis E (2000). Contributions of behavioral science to alcohol research: understanding who is at risk and why [In Process Citation]. Exp Clin Psychopharmacol 8: 264-270.

Green AI, Zimmet SV, Strous RD, Schildkraut JJ (1999). Clozapine for comorbid substance use disorder and schizophrenia: do patients with schizophrenia have a reward-deficiency syndrome that can be ameliorated by clozapine? Harvard Rev Psychiatry 6: 287-296.

Hutchison KE, LaChance $H$, Niaura R, Bryan AD, Smolen A (2002a). The DRD4 VNTR poylmorphism influences reactivity to smoking cues. J Abnorm Psychol 111: 134-143.

Hutchison KE, McGeary J, Smolen A, Bryan AD, Swift RM (2002b). The DRD4 VNTR polymorphism moderates craving after alcohol consumption. Health Psychol 21: 139-146.

Hutchison KE, McGeary J, Smolen A, Wooden A (2001). Craving after alcohol consumption: olanzapine and the DRD4 VNTR polymorphism. Alcoholism: Clin Exp Res 25: 66a.

Hutchison KE, Stallings M, McGeary J, Bryan A. Population stratification in the candidate gene study: fatal threat or red herring? Psychol Bull (in press).

Johnson BA, Roache JD, Ait-Daoud N, Zanca NA, Velazquez M (2002). Ondansetron reduces the craving of biologically predisposed alcoholics. Psychopharmacology (Berl) 160: 408-413.

Kapur S, Zipursky RB, Jones C, Wilson AA, DaSilva JD, Houle S (1997). Cyproheptadine: a potent in vivo serotonin antagonist [letter]. Am J Psychiatry 154: 884.

Lench N, Stanier P, Williamson R (1988). Simple non-invasive method to obtain DNA for gene analysis. Lancet 1: 1356-1358.

Lichter JB, Barr CL, Kennedy JL, Van Tol HH, Kidd KK, Livak KJ (1993). A hypervariable segment in the human dopamine receptor D4 (DRD4) gene. Hum Mol Genet 2: 767-773.

Martin CS, Earleywine M, Musty RE, Perrine MW, Clinic PU, Swift RM (1993). Development and validation of the Biphasic Alcohol Effects Scale. Alcoholism: Clin Exp Res 17: 140-146.

Modell JG, Mountz JM (1995). Focal cerebral blood flow change during craving for alcohol measured by SPECT. J Neuropsychiatry Clin Neurosci 7: 15-22. 
Modell JG, Mountz JM, Glaser FB, Lee JY (1993). Effect of haloperidol on measures of craving and impaired control in alcoholic subjects. Alcohol Clin Exp Res 17: 234-240.

Robinson TE, Berridge KC (1993). The neural basis of drug craving: an incentive-sensitization theory of addiction. Brain Res Brain Res Rev 18: 247-291.

Sander T, Harms H, Dufeu P, Kuhn S, Rommelspacher H, Schmidt LG (1997). Dopamine D4 receptor exon III alleles and variation of novelty seeking in alcoholics. Am J Med Genet 74: 483-487.

Skinner HA, Allen BA (1982). Alcohol dependence syndrome: measurement and validation. J Abnorm Psychol 91: 199-209.

Sobell LC, Sobell MB (1980). Convergent validity: an approach to increasing confidence in treatment outcome conclusions with alcohol and drug abusers. In: Sobell LC, Sobell MB, Ward E (eds) Evaluating Alcohol and Drug Abuse Treatment Effectiveness: Recent Advances pp 177-183, Pergamon Press: New York.
Swift RM (1999). Medications and alcohol craving. Alcohol Res Health 23: 207-213.

Swift RM, Whelihan W, Kuznetsov O, Buongiorno G, Hsuing H (1994). Naltrexone-induced alterations in human ethanol intoxication. Am J Psychiatry 151: 1463-1467.

Wacholder S, Rothman N, Caporaso N (2000). Population stratification in epidemiologic studies of common genetic variants and cancer: quantification of bias. J Natl Cancer Inst 92: $1151-1158$.

Watson PE (1989). Total body water and blood alcohol levels: updating the fundamentals. In: Crow KE, Batt RD (ed) Human Metabolism of Alcohol pp 41-56, CRC Press: Boca Raton, FL.

Wise RA (1988). The neurobiology of craving: implications for the understanding and treatment of addiction. J Abnorm Psychol 97: 118-132. 